\title{
Key proteins involved in insulin vesicle exocytosis and secretion (Review)
}

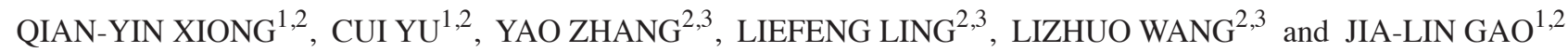 \\ ${ }^{1}$ Department of Endocrinology and Genetic Metabolism, Yijishan Hospital of Wannan Medical College, Wuhu, Anhui 241002; \\ ${ }^{2}$ Anhui Province Key Laboratory of Biological Macro-molecules Research, Wannan Medical College, Wuhu, Anhui 242001; \\ ${ }^{3}$ Department of Biochemistry and Molecular Biology, Wannan Medical College, Wuhu, Anhui 241001, P.R. China
}

Received May 12, 2016; Accepted November 11, 2016

DOI: $10.3892 /$ br.2017.839

\begin{abstract}
In vivo insulin secretion is predominantly affected by blood glucose concentration, blood concentration of amino acids, gastrointestinal hormones and free nerve functional status, in addition to other factors. Insulin is one of the most important hormones in the body, and its secretion is precisely controlled by nutrients, neurotransmitters and hormones. The insulin exocytosis process is similar to the neurotransmitter release mechanism. There are various types of proteins and lipids that participate in the insulin secretory vesicle fusion process, such as soluble $\mathrm{N}$-ethylmaleimide-sensitive factor attachment protein receptor (SNARE) protein, Ras-related proteins and vacuolar-type $\mathrm{H}^{+}$-ATPase (V-ATPase). Notably, the SNARE protein is the molecular basis of exocytotic activity. In the current review, the role of the vesicle membrane proteins (synaptobrevins, vesicle associated membrane proteins and target membrane proteins) and auxiliary proteins (Rab proteins and Munc-18 proteins) in vesicle fusion activity were summarized. A summary of these key proteins involved in insulin granule secretion will facilitate understanding of the pathogenesis of diabetes.
\end{abstract}

\section{Contents}

1. Introduction

2. Role of SNARE proteins in exocytosis

Correspondence to: Dr Jia-Lin Gao, Department of Endocrinology and Genetic Metabolism, Yijishan Hospital of Wannan Medical College, 2 Zheshan Road, Wuhu, Anhui 241002, P.R. China E-mail: jialing.gao@yahoo.com

Mrs. Lizhuo Wang, Department of Biochemistry and Molecular Biology, Wannan Medical College, 22 West Wenchang Road, Wuhu, Anhui 241001, P.R. China

E-mail: lizhuo.wang@yahoo.com

Key words: insulin secretion, secretory vesicle, exocytosis, regulation
3. Role of vacuolar-type $\mathrm{H}^{+}$-ATPase (V-ATPase) in exocytosis

4. Other proteins that participate in insulin secretory granule exocytosis

5. Regulation of insulin secretory granule exocytosis

6. Conclusion and future perspectives

\section{Introduction}

Insulin is a peptide hormone, which is stored in cells in large dense-core secretory granules and secreted by the islets $\beta$-cells, released through granule exocytosis, upon stimulation (1). Exocytosis, a key process in insulin secretion, includes two steps; intracellular insulin secretory vesicle transport and insulin secretory vesicle fusion with the plasma membrane (2). Fusion of the insulin secretory vesicle and the plasma membrane involves the formation of an array of soluble $\mathrm{N}$-ethylmaleimide-sensitive factor attachment protein receptor (SNARE) complex proteins (3), as well as many other important proteins. The trafficking and fusion of secretory vesicles involve multiple steps and complex proteins interactions, and are subject to strict regulation. Currently, the specific mechanisms of the biosynthesis and maturation of insulin particles, as well as the specific mechanisms by which it is released from cells remains unclear. Therefore, understanding the molecular secretion process will improve the understanding of the pathogenesis of diabetes mellitus (DM) and provide information for the treatment of diabetes. The current study aims to demonstrate the mechanism of insulin secretory vesicle exocytosis and the regulation of insulin secretion.

\section{Role of SNARE proteins in exocytosis}

Types and construction of SNARE proteins. SNAREs are content-rich transmembrane proteins. The SNARE protein is a transmembrane protein family, which is located in cell organelles and vesicles. There are $>30$ types of SNARE family protein in mammalian cells, each of which is in a separate sub cellular component. Although the size of SNAREs varies, their structure contains a common SNARE domain, which consists of 60-70 amino acids, in a coiled-coil structure containing seven repeats. This domain determines all the characteristics 
of SNAREs and mediates the formation of SNARE complexes; therefore it is significant to SNARE function. In addition, SNAREs contain two domains as follows: One that is located in the transmembrane domain of the C-terminal end, responsible for anchoring the bubble film (the fusion of vesicle membrane proteins and target membrane proteins) of SNAREs in the target capsule. The synaptobrevin protein promotes the SNARE zipper-like binding reaction through the transmembrane domain of the C-terminal, resulting in fusion of the vesicles. The second is the regulating domain of the N-terminal, which is responsible for regulating SNARE activity (4).

There are two classifications of SNAREs; the first is based on the different subcellular localization, divided into vesicle (v)-SNAREs and target ( $\mathrm{t}$ )-SNAREs from the target membrane. The second classification is based on the amino acid located in the center of the SNARE domain, arginine (Arg) or glutamine (Glu), which is divided into R-SNAREs and Q-SNAREs, respectively (5). Under normal circumstances, there are more t-SNAREs than Q-SNAREs and more v-SNAREs than R-SNAREs (4). In addition, Q-SNAREs are further divided into Qa-SNAREs (syntaxin), Qb-SNAREs and Qc-SNAREs (5).

$t$-SNARES. t-SNARE is distributed on the target membrane, including the syntaxin and SNAP-25 families (5). Various studies have confirmed that syntaxin-1a and synaptosome associated protein 25 (SNAP-25) have formed a specific t-SNARE two element complex (6). In vitro studies have shown that the interaction of the syntaxin-1a/SNAP-25 complex with vesicle-associated membrane protein 2 (VAMP2) produces a coiled coil, which provides sufficient energy for driving the fusion reaction (4). Prior to formation of the SNARE complex, Sec1/Munc18 (SM) protein and Qa-SNARE binding occurred, so that the space between membranes was closed. When membrane fusion begins, the SM protein binds with the N-terminal end of the Qa-SNARE, so that it is exposed to the SNARE domain (7). First, the Qa-SNARE binds with the Qc-SNARE and Qb-SNARE to form a three element complex. When the vesicle membrane docks in the target membrane, the three element complex of the SNAREs complex and R-SNARE forms a specific four element complex (loose complex). Subsequently, assembly of the SNAREs core complex. SNAREs connect the vesicle membrane and cell membrane via three molecular structures. One of which is composed of synaptobrevin and syntaxin proteins, and SNAP-25 forms the two other molecular structures. The SNAREs core complex is composed of relational twisting of numerous parallel protein spiral structures, forming a leucine zipper embedded structure, the leucine zipper enables the connection of two domains between adjacent vesicles and cell membranes. The SNARE transmembrane domain is anchored during the fusion of the two films, while t-SNARE and v-SNARE mediate membrane fusion to connect t-SNARE and v-SNARE at a certain position (8). The t-SNARE complex contains a Glu relatively conserved sequence, which is positioned in the center of the helix bundle (termed Q-SNARE protein). The v-SNARE contributes an Arg sequence (termed R-SNARE protein) and is also in this position. Certain proteins [such as RAB3A, member RAS oncogene family (Rab3A)] coexist on the vesicle membrane and the target membrane, establishing the Q-R-SNARE classification model, which is an increasingly prevalent model. This model is based on two conserved amino acid sequences of the cytoplasmic domain of SNARE protein, Glu (Q) is a conserved sequence, $\operatorname{Arg}(\mathrm{R})$ is another conserved sequence. Those cytoplasmic domain contains Glu conserved sequence of SNARE protein (such as Syntaxin, SNAP25) and is classified as Q-SNARE, whereas SNARE protein (such as VAMP and Rab3A) containing an Arg conserved sequence is referred to as R-SNARE. The next R-SNARE and Qa-SNARE transmembrane domain rearrangement forms cis-SNAREs, prompting membrane distal binding and, thus, the formation of fusion pores and fusion pore expansion, which releases the material from inside the vesicle (9).

$v$-SNAREs (the role of VAMP proteins in exocytosis). VAMP is a v-SNARE anchored to synaptic vesicles and secretory granules. V-SNARE is distributed on the vesicle membrane, along with synaptobrevin/VAMPs and associated proteins (10). The v-SNARE complex is formed during a membrane fusion process, which requires a series of molecules, such as synaptobrevins, syntaxins and SNAREs core proteins (such as synaptic-associated proteins) to achieve synergy. According to recent analysis of VAMP (11), the detailed proteomic characterizations of granule secretory vesicles were identified. There are four identified VAMPs, including VAMP2, VAMP3, VAMP7 and VAMP8. VAMP2, VAMP3 and VAMP8 are known to participate in exocytosis in various cell types and to form a complex substance with syntaxin 4/SNAP-23 (12). VAMP2, a v-SNARE granule protein, is important; it is a structural protein of the synaptic vesicle membrane, which is closely associated with all of the neurons and endocrine cells, which are closely associated with neurotransmitter, hormone release and synaptic plasticity.

For example, in the compound of syntaxin 1a, following dissociation, VAMP2 or SNAP-25 toxins cause $\beta$-cell insulin secretion. Notably, in vitro studies have shown that the interaction of the syntaxin 1/SNAP-25 complex and VAMP2 produces a spiral beam, which provides enough energy to drive the fusion reaction (13). VAMP7 is predominantly located in the inner body, lysosomes and the cell membrane, which is involved in vesicle transport between the inner body and the lysosome. VAMP5, located in the cell membrane, is highly expressed in muscle cells, and is associated with the cell membrane and vesicle structure, and myotubes. VAMP5 is usually considered to be an adjustable vesicle that mediates docking and fusion of vesicles to target membranes, and is important for membrane transport of skeletal and cardiac muscle. VAMP8 is located in the cell membrane, and early and late endosomes found in the trans Golgi network and other organelles, suggest its extensive role. A previous study indicates that VAMP8 mediated endocytosis of glucose transporter type 4 (14).

$R$-SNARE (synaptobrevin). The SNARE protein is formed according to the zipper hypothesis; a zipper exists at the interface of the v-/t-SNARE interaction and, therefore, is equivalent to providing a fusion reaction to an inward tension (15). t-SNARE is distributed on the target membrane, including the syntaxin and SNAP-25 families. v-SNAREs are distributed on the vesicle membrane, and include synaptobrevins/VAMPS 


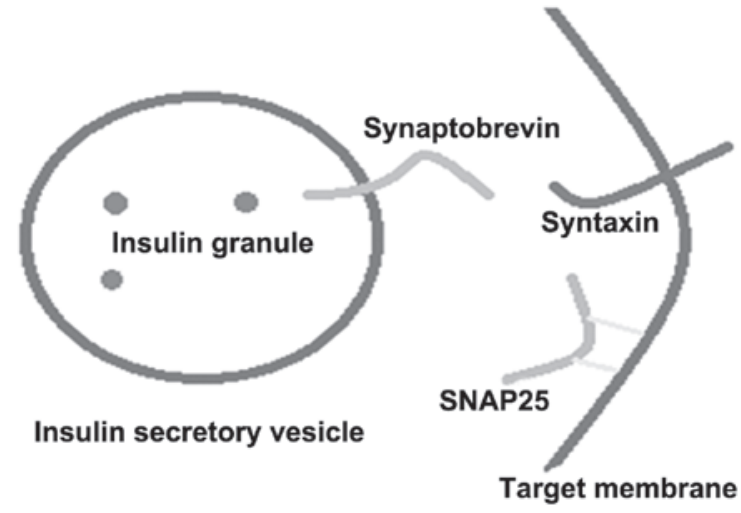

Figure 1. Role of synaptobrevin proteins in exocytosis. SNAP-25, synaptosome associated protein 25 .

and associated proteins. Synaptobrevins, syntaxins and SNAP-25 form a stable melamine according to the proportion of 1:1:1 (Fig. 1) (4). The SNARE motif in the three classes of the SNARE protein is formed in parallel with the $\alpha$ helical region of the $\mathrm{N}$-motif, which forms the core complex (N-ethylmaleimide-sensitive factor; NSF). Under the assistance of the $\alpha / \beta$-soluble $N$-ethylmaleimide-sensitive attachment proteins (SNAPs), the hydrolysis of ATP results in SNARE polymer dissociation (16).

\section{Role of vacuolar-type $\mathbf{H}^{+}$-ATPase (V-ATPase) in exocytosis}

Molecular characteristic of V-ATPase. A protein that is widely distributed in the eukaryotic cell membrane and the cell membrane of the cell membrane, which is associated with a type of V-type $\mathrm{H}^{+}$ATPase. The structure of V-ATPase is composed of two subunits, V1 and V0 in the cytoplasm. The former provides a channel for $\mathrm{H}^{+}$and the latter decomposes ATP, and provides energy for the transfer of $\mathrm{H}^{+}$against the concentration gradient. V0 and V1 are only involved in polymeriztion (17). Tonoplast V-ATPase is a particularly important type of proton pump, involved in ion homeostasis in the cytoplasm and in cell metabolism. This enzyme is a large multimeric enzyme composed of cytoplasmic and membrane surface V1 and V0 complexes. The V1 domain, located in the cytoplasm, contains eight subunits (A3, B3, C, D, E, F, $\mathrm{G} 2$ and $\mathrm{H}$ ), which are responsible for the hydrolysis of ATP, with subunits A and B being the most important. Subunit A catalyzes the hydrolysis of ATP (subunit B is not conserved in the glycine rich region; therefore, has no catalytic function, but has the ability to regulate). ATP is involved in the hydrolysis of the A3 and B subunits, which function in a similar way to the F-ATPase $\beta$ and $\alpha$ subunits (18). There are three copies of each of the A and B subunits, to form the six polymer units. The A subunit is the catalytic subunit of the hydrolysis of ATP and the B subunit is a non-catalytic subunit, which is a site of ATP binding. Certain residues of the A subunit are essential for enzyme activity, such as E286, which is involved in proton uptake. Negatively charged phosphate groups, located in the glycine rich region, may be associated with the ATP phase of K263. The B subunit is the regulatory subunit, and each of the six polymer units contains three sites for the catalytic hydrolysis of ATP $(19,20)$. The V0 domain is composed of five subunits (a, c, c", d and e), which are responsible for proton transport.

Function of V-ATPase in exocytosis. V-ATPase activity in neuronal synaptic vesicles produced a large number of proton electrochemical gradients (21). This electrochemical proton gradient is a low $\mathrm{pH}$ condition for the accumulation of neurotransmitter in the synaptic vesicles or catecholamine in the granules of the granule (22). The V0 region of V-ATPase was found to interact with proteins involved in the extracellular region of the protein (Fig. 2). The interaction of V0 and VAMP2 may be expressed in the cis complex of the same insulin secretory vesicles (15). The interaction between $\mathrm{V}$-ATPase and syntaxin-1 is also important for the regulation of the cell, and these interactions are regulated by calmodulin and $\mathrm{Ca}^{2+}(21)$.

\section{Other proteins participate in insulin secretory granules exocytosis}

SM proteins participate in insulin secretory granule exocytosis. The majority of transport pathways require a SNARE protein complex that mediates the fusion of transport vesicles and target membranes. Simultaneously, another protein family, SM proteins, is significant to vesicle transport (4). The SM protein is a type of hydrophilic protein, which is composed of $\sim 600$ amino acids. The homology between the family members is high. The spatial structure of the family is roughly the 'bow' structural protein, which is closely associated with secretion (5). It has been identified that integration of the cell membrane in the vesicle transport pathway requires SNARE protein family involvement (4). The family is predominantly composed of two subtypes of the target membrane, t-SNARE and v-SNARE vesicles. $\mathrm{v}$ - and t-SNAREs are assembled into SNARE complexes, which is promoted by the fusion of the vesicle membrane and the target membrane, thus is key in the membrane transport process. However, v- and t-SNARE do not spontaneously assemble into a complex, the assembly of the anterior vesicle anchored target membrane and the SNARE complex requires the involvement of other proteins. This includes SM protein family members, which are significant in SNARE-mediated membrane fusion and vesicle trafficking events $(3,22)$. Previous studies suggest that SM proteins may regulate the process of vesicle anchoring to the target membrane, and may also be involved in the initiation, fusion and other steps in the regulation (23). The interaction between SM proteins and the SNARE complex has been identified to occur via four primary mechanisms (24) as follows: i) The SM proteins bind to the closed conformation of the syntaxin protein. This combination makes the syntaxin-1 and munc18-1 more stable in the body relative to their respective monomer forms (6). ii) Certain SM proteins interact in other ways with the syntaxin protein family; the SM proteins combine with the $\mathrm{N}$ terminal sequence of the conserved syntaxin protein, which is termed the open state. This interaction is more common than the aforementioned interaction $(6,25)$. iii) The direct combination of SM proteins and the SNARE complex. SM proteins directly bind to the SNARE complex, which 
A

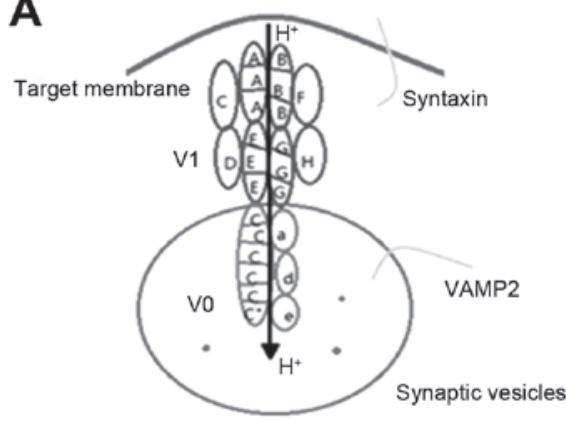

C

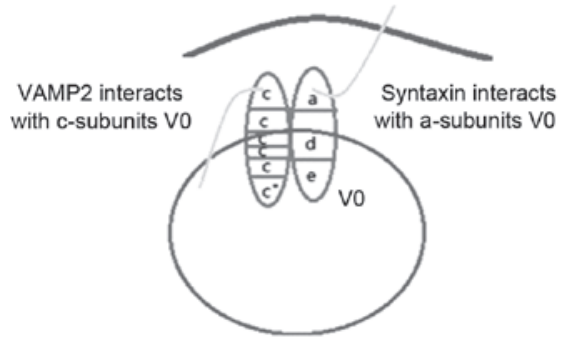

B

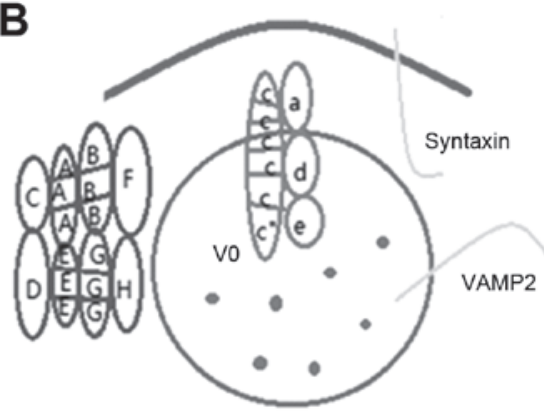

D

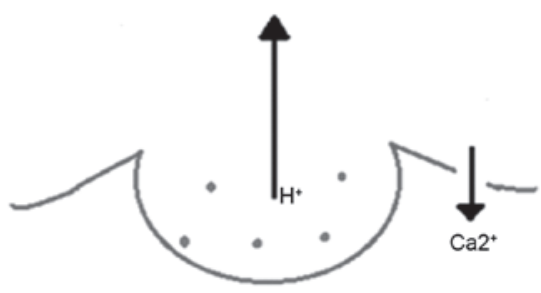

Figure 2. Role of vacuolar ATPase in exocytosis: (A) Loading; (B) ready for exocytosis; (C) docking and priming; and (D) membrane fusion. VAMP2, vesicle-associated membrane protein 2.

A

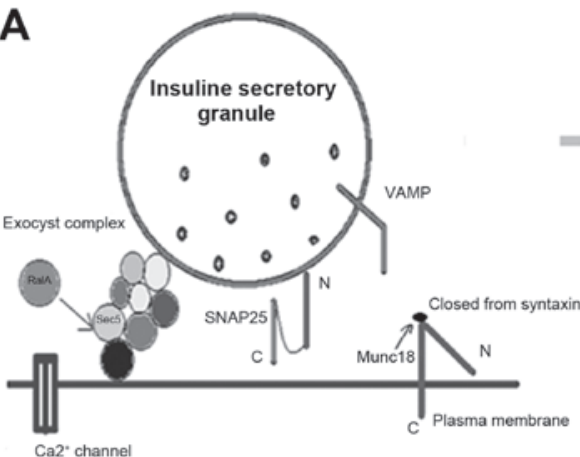

C

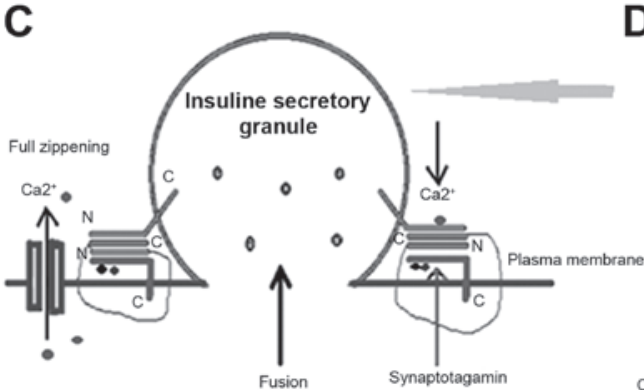

B

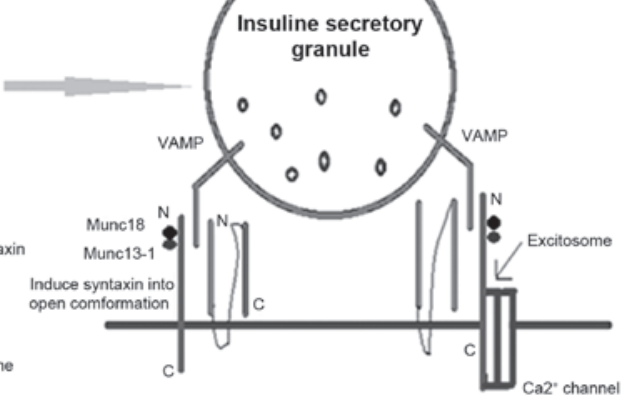

D

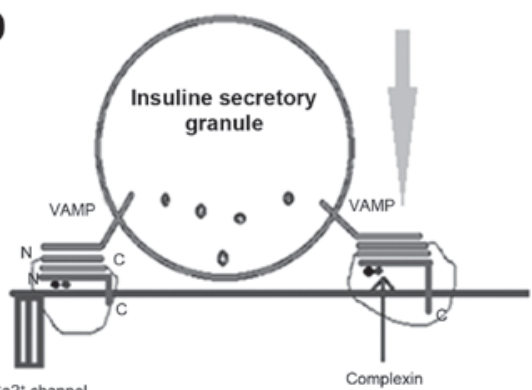

Figure 3. Steps of exocytosis. (A) Secretory granule tethering to the plasma membrane. (B) Priming. (C) Fully primed. (D) Fusion. VAMP, vesicle-associated membrane protein 2; SNAP-25, synaptosome associated protein 25 .

indicates that the SM protein is involved in the regulation of the fusion of vesicles and target membranes (26). iv) A small number of SM proteins do not interact directly with syntaxin, but first bind to other soluble proteins and form a complex, and subsequently bind to the syntaxin protein. Numerous studies demonstrate that the correct assembly of the SNARE complex predominantly depends on the regulation of SM proteins, and the binding of SM proteins and the SNARE complex ensures the stability of the SNARE complex (5).

Rab proteins and insulin secretory granule exocytosis. Rab protein is the largest family of the small molecule guanosine-5'-triphosphate (GTP) binding protein family (small GTP-binding proteins). Composed of 200 amino acids, 
it comprises a conserved $\mathrm{G}$ domain with highly variable $\mathrm{N}$ and $\mathrm{C}$ ends. The Rab protein serves as a vesicle trafficking molecular switch, and its upstream regulator and downstream effector specific interactions, coupled with the GTP binding and hydrolysis process, are involved in the different stages of vesicular transport (27). The Rab protein is ubiquitous in vesicular transport components contributing to the basic functions, as well as the regulatory functions of vesicle transport. Rab proteins facilitate vesicle aggregation close to the target membrane, triggering SNARE to release inhibiting factor. Rab protein is a GTP binding protein and Rab-GTP guides transport vesicles from the donor area to the target area of the plasma membrane, with assistance from SNAP25. The Rab-GTP complex combines with the vesicle SNAP receptor (t-SNARE) to form the anterior fusion complex. The Rab protein hydrolyzes GTP to generate guanosine-5'-triphosphate (GDP), and the Rab-GDP complex separates from the vesicles and binds with the GDP dissociation inhibitor (GDI) in the cytoplasm. This is then transferred to the plasma membrane of the donor site. Rab-GDP separates from GDI and combines with guanine nucleotide exchange factor (GEF), stimulating GTP replacement of GDP. Rab-GTP may then recombine with transport vesicles; simultaneously, Three classes of SNAP combine with the anterior fusion complex and combines with the ATP enzyme, NSF. NSF hydrolyses ATP, which triggers the fusion of vesicles and target membranes. Transport vesicles are only able to form when they contain specific Rabs and SNAREs. Rab proteins connect or activate specific effectors, and regulate vesicle transport during vesicle formation, transport, adhesion, anchoring, and fusion at different transport phases (28). Furthermore, vesicle formation requires interactions between multiple proteins, such as ADP-ribosylation factor GTPases, coatmer protein subunits, cohesion and grid proteins. Thus, the Rab protein is essential for vesicle formation (29). In the cytosol, vesicular transport must occur over a long distance. Rab proteins interact with actin and tubulin in the cytoskeleton to regulate vesicle transport. For example Rab-6 (Rabkinesin-6), a kinesin effector, is a member of the kinesin family; it has the traditional structural characteristics of kinesin. Rabkinesin-6 interacts with Rab6-GTP, and uses the microtubule-based cytoskeleton to guide vesicle transport (30). The Rab protein interacts with adhesion factors during the formation of vesicles (i.e., in the absorption of SNARE to transport vesicles). Rab protein interactions occur instantaneously by the same adhesion factor, in any order, during the two stages (formation and adhesion) of vesicular transport and have an important role (9). In the target membrane vesicles are anchored by pairing of $\mathrm{v}$ - and $\mathrm{t}$-SNAREs, and this pairing provides specificity for the membrane fusion reactions. The Rab protein interacts with adhesion factors and other effectors to promote the pairing of $\mathrm{v}$ - and t-SNAREs, and subsequently promotes vesicle fusion with the target membrane (27).

\section{Regulation of insulin secretory granule exocytosis}

Peptide and protein hormones are stored in and released by the secretory vesicles. Large secretory vesicles are generated by the Golgi to form a network structure. Subsequently, a series of steps result in exocytosis as follows: Recruitment (the release of secretory vesicles to the cell membrane), anchoring, priming (the maturation of the vesicles), and the final vesicle membrane and cell membrane fusion. The inclusions are then released into the extracellular fluid through the fusion pore (Fig. 3).

There are two types of factors regulating insulin secretion: A class of nutrients, such as glucose, and the neurotransmitters and hormones. Islet $\beta$-cells integrate these two kinds of signal functions of regulating factors, and adjust their insulin levels in steady state. At the molecular level, the insulin secretory vesicle exocytotic mechanism and its regulation include two parts: One is the proximal step, which involves adjusting the level of intracellular second messenger substances. The second is the terminal adjustment steps. In the proximal step, metabolism of glucose and other nutrients, via alterations of the cytoplasm ATP/ADP ratio, closes the ATP-sensitive potassium $\left(\mathrm{K}^{+}\right)$channel resulting in membrane depolarization-induced calcium $\left(\mathrm{Ca}^{2+}\right)$ channel opening. Increased intracellular $\mathrm{Ca}^{2+}$ ion levels stimulate the release of insulin. Elevated intracellular $\mathrm{Ca}^{2+}$ leads to a rise of ATP/ADP in the cytoplasm, and the $\mathrm{K}^{+}$channels close causing membrane depolarization, $\mathrm{Ca}^{2+}$ influx and insulin granular exocytosis (31). Glucose-dependent insulinotropic polypeptide (32) and glucagon-like peptide 1 (GLP-1) (33) act on membrane receptors to generate cytoplasmic cAMP and regulate insulin secretion. The insulin pathway is initiated by the binding of insulin to the receptor on the target tissue. Under physiological conditions, insulin action on the insulin receptor (INSR) of the target cell membrane combined with the $\alpha$ subunit of the receptor, results in multiple $\beta$ subunit tyrosine phosphorylation of the INSR substrate, and subsequently combines with the INSR substrates (IRS-1 and IRS-2). The tyrosine protein substrates are phosphorylated by tyrosine kinases of the INSR (34). Phosphorylated IRS-1 and IRS-2 interact with the p85 subunit of phosphoinositide 3-kinase (PI3K), to bring it closer to the plasma membrane, leading to the generation of phosphatidylinositol $(3,4,5)$-trisphosphate $(35)$. Through a series of signal transduction pathways, inhibition of glycogen synthase kinase 3 (GSK-3) promotes glycogen synthase (GS) phosphorylation, improves its activity, promotes tissue glycogen synthesis and decreases the peripheral blood glucose concentration (34).

\section{Conclusion and future perspectives}

The current review introduces the proteins that are involved in insulin secretory vesicle exocytosis. It is these proteins that pull the vesicle membrane and the cell membrane together, resulting in the exocytosis process. Membrane fusion failure may be associated with the occurrence of diabetes; therefore, the specific mechanisms of membrane fusion are considered to be significant in the prevention of diabetes. Currently, the understanding of the various aspects of membrane fusion remains hypothetical. Understanding the process of membrane fusion and the underlying molecular mechanisms will markedly promote the development of biology, as well as lay a solid foundation for disease treatment. Furthermore, the pathogenesis of DM, particularly the early mechanisms, is unclear. Inhibition of insulin secretory granule exocytosis is the important pathological manifestation in the early stage of DM development. Therefore, further in-depth investigation of DM and disordered early insulin secretion, and timely clarification 
of pathophysiologic mechanisms are considered to be particularly urgent. In biology and morphology, secretory vesicles and lysosomes possess a high degree of homology and similarity. In addition, lysosome membrane proteins, and the association between insulin secretory vesicles and their role in exocytosis have not yet been investigated in detail. Due to the biological homology of lysosomes and vesicles, further investigations are required to establish whether lysosomal membrane proteins have an important role in the formation of insulin secretion vesicles and insulin secretion. Thus, their involvement in the early development of DM and insulin secretion disorder requires further investigation.

\section{Acknowledgements}

The study was supported by the National Natural Science Foundation of China (grant nos. 81200632 and 81471002), the Natural Science Foundation of Anhui, China (grant no. 1308085QH134), and the Introduction of Talents Foundation of Yijishan Hospital (grant no. YR201104).

\section{References}

1. Liu T, Li H, Gounko NV, Zhou Z, Xu A, Hong W and Han W: Detection of insulin granule exocytosis by an electrophysiology method with high temporal resolution reveals enlarged insulin granule pool in BIG3-knockout mice. Am J Physiol Endocrinol Metab 307: E611-E618, 2014.

2. Li J, Cantley J, Burchfield JG, Meoli CC, Stöckli J, Whitworth PT, Pant H, Chaudhuri R, Groffen AJ, Verhage M, et al: DOC2 isoforms play dual roles in insulin secretion and insulin-stimulated glucose uptake. Diabetologia 57: 2173-2182, 2014.

3. Jewell JL, Oh E and Thurmond DC: Exocytosis mechanisms underlying insulin release and glucose uptake: Conserved roles for Munc18c and syntaxin 4. Am J Physiol Regul Integr Comp Physiol 298: R517-R531, 2010.

4. Südhof TC and Rothman JE: Membrane fusion: Grappling with SNARE and SM proteins. Science 323: 474-477, 2009.

5. Hong W: SNAREs and traffic. Biochim Biophys Acta 1744: 493-517, 2005.

6. Jahn R: Sec1/Munc18 proteins: Mediators of membrane fusion moving to center stage. Neuron 27: 201-204, 2000.

7. Ulloa F, Gonzàlez-Juncà A, Meffre D, Barrecheguren PJ, Martínez-Mármol R, Pazos I, Olivé N, Cotrufo T, Seoane J and Soriano E: Blockade of the SNARE protein syntaxin 1 inhibits glioblastoma tumor growth. PLoS One 10: e0119707, 2015.

8. Alonso-Curbelo D, Riveiro-Falkenbach E, Pérez-Guijarro E, Cifdaloz M, Karras P, Osterloh L, Megías D, Cañón E, Calvo TG, Olmeda D, et al: RAB7 controls melanoma progression by exploiting a lineage-specific wiring of the endolysosomal pathway. Cancer Cell 26: 61-76, 2014

9. Torii S, Takeuchi T, Nagamatsu S and Izumi T: Rab27 effector granuphilin promotes the plasma membrane targeting of insulin granules via interaction with syntaxin 1a. J Biol Chem 279: 22532-22538, 2004.

10. Lin RC and Scheller RH: Mechanisms of synaptic vesicle exocytosis. Annu Rev Cell Dev Biol 16: 19-49, 2000.

11. Borisovska M, Zhao Y, Tsytsyura Y, Glyvuk N, Takamori S, Matti U, Rettig J, Südhof T and Bruns D: v-SNAREs control exocytosis of vesicles from priming to fusion. EMBO J 24: 2114-2126, 2005.

12. Müller HK, Kragballe M, Fjorback AW and Wiborg O: Differential regulation of the serotonin transporter by vesicleassociated membrane protein 2 in cells of neuronal versus non-neuronal origin. PLoS One 9: e97540, 2014.

13. Nagamatsu S, Nakamichi Y, Watanabe T, Matsushima S, Yamaguchi S, Ni J, Itagaki E and Ishida H: Localization of cellubrevin-related peptide, endobrevin, in the early endosome in pancreatic beta cells and its physiological function in exo-endocytosis of secretory granules. J Cell Sci 114: 219-227, 2001.
14. Antonin W, Holroyd C, Tikkanen R, Höning S and Jahn R: The R-SNARE endobrevin/VAMP-8 mediates homotypic fusion of early endosomes and late endosomes. Mol Biol Cell 11: 3289-3298, 2000.

15. Forgac M: Vacuolar ATPases: Rotary proton pumps in physiology and pathophysiology. Nat Rev Mol Cell Biol 8: 917-929, 2007.

16. Toei M, Saum R and Forgac M: Regulation and isoform function of the V-ATPases. Biochemistry 49: 4715-4723, 2010.

17. Morel N: Neurotransmitter release: The dark side of the vacuolar$\mathrm{H}^{+}$ATPase. Biol Cell 95: 453-457, 2003.

18. Poëa-Guyon S, Amar M, Fossier P and Morel N: Alternative splicing controls neuronal expression of v-ATPase subunit a1 and sorting to nerve terminals. J Biol Chem 281: 17164-17172, 2006.

19. Camacho M, Machado JD, Montesinos MS, Criado M and Borges R: Intragranular $\mathrm{pH}$ rapidly modulates exocytosis in adrenal chromaffin cells. J Neurochem 96: 324-334, 2006.

20. Di Giovanni J, Boudkkazi S, Mochida S, Bialowas A, Samari N, Lévêque C, Youssouf F, Brechet A, Iborra C, Maulet Y, et al: V-ATPase membrane sector associates with synaptobrevin to modulate neurotransmitter release. Neuron 67: 268-279, 2010.

21. Ahmad A, Khundmiri SJ, Pribble F, Merchant ML, Ameen M, Klein JB, Levi M and Lederer ED: Role of vacuolar ATPase in the trafficking of renal type IIa sodium-phosphate cotransporter. Cell Physiol Biochem 27: 703-714, 2011.

22. Kwan EP and Gaisano HY: Rescuing the subprime meltdown in insulin exocytosis in diabetes. Ann N Y Acad Sci 1152: 154-164, 2009.

23. Kwan EP, Xie L, Sheu L, Nolan CJ, Prentki M, Betz A, Brose N and Gaisano HY: Munc13-1 deficiency reduces insulin secretion and causes abnormal glucose tolerance. Diabetes 55: 1421-1429, 2006.

24. Zhu D, Zhang Y, Lam PP, Dolai S, Liu Y, Cai EP, Choi D, Schroer SA, Kang Y, Allister EM, et al: Dual role of VAMP8 in regulating insulin exocytosis and islet $\beta$ cell growth. Cell Metab 16: 238-249, 2012

25. Mandic SA, Skelin M, Johansson JU, Rupnik MS, Berggren PO and Bark C: Munc18-1 and Munc18-2 proteins modulate beta-cell $\mathrm{Ca}^{2+}$ sensitivity and kinetics of insulin exocytosis differently. J Biol Chem 286: 28026-28040, 2011.

26. Parsaud L, Li L, Jung CH, Park S, Saw NM, Park S, Kim MY and Sugita S: Calcium-dependent activator protein for secretion 1 (CAPS1) binds to syntaxin-1 in a distinct mode from Munc13-1. J Biol Chem 288: 23050-23063, 2013.

27. Brunner Y, Couté Y, Iezzi M, Foti M, Fukuda M, Hochstrasser DF, Wollheim CB and Sanchez JC: Proteomics analysis of insulin secretory granules. Mol Cell Proteomics 6: 1007-1017, 2007.

28. Jordens I, Marsman M, Kuijl C and Neefjes J: Rab proteins, connecting transport and vesicle fusion. Traffic 6: 1070-1077, 2005.

29. Iezzi M, Escher G, Meda P, Charollais A, Baldini G, Darchen F, Wollheim CB and Regazzi R: Subcellular distribution and function of Rab3A, B, C, and D isoforms in insulin-secreting cells. Mol Endocrinol 13: 202-212, 1999.

30. Waselle L, Coppola T, Fukuda M, Iezzi M, El-Amraoui A, Petit $\mathrm{C}$ and Regazzi R: Involvement of the Rab27 binding protein Slac2c/MyRIP in insulin exocytosis. Mol Biol Cell 14: 4103-4113, 2003.

31. Yang SN and Berggren PO: Beta-cell $\mathrm{CaV}$ channel regulation in physiology and pathophysiology. Am J Physiol Endocrinol Metab 288: E16-E28, 2005.

32. Gremlich S, Porret A, Hani EH, Cherif D, Vionnet N, Froguel P and Thorens B: Cloning, functional expression, and chromosomal localization of the human pancreatic islet glucose-dependent insulinotropic polypeptide receptor. Diabetes 44: 1202-1208, 1995.

33. Thorens B: Expression cloning of the pancreatic beta cell receptor for the gluco-incretin hormone glucagon-like peptide 1 . Proc Natl Acad Sci USA 89: 8641-8645, 1992.

34. Dominguez V, Raimondi C, Somanath S, Bugliani M, Loder MK, Edling CE, Divecha N, da Silva-Xavier G, Marselli L, Persaud SJ, et al: Class II phosphoinositide 3-kinase regulates exocytosis of insulin granules in pancreatic beta cells. J Biol Chem 286: 4216-4225, 2011.

35. Guo L, Li Q, Wang W, Yu P, Pan H, Li P, Sun Y and Zhang J: Apelin inhibits insulin secretion in pancreatic beta-cells by activation of PI3-kinase-phosphodiesterase 3B. Endocr Res 34: $142-154,2009$. 